\title{
Yves Pauwels, Aux marges de la règle. Essai sur les ordres d'architecture à la Renaissance
}

Wavre, Mardaga, 2008, 191 pages

Denyse Rodriguez Tomé

\section{OpenEdition}

\section{Journals}

Édition électronique

URL : http://journals.openedition.org/dht/273

DOI : $10.4000 /$ dht.273

ISSN : 1775-4194

Éditeur :

Centre d'histoire des techniques et de l'environnement du Cnam (CDHTE-Cnam), Société des élèves du CDHTE-Cnam

\section{Édition imprimée}

Date de publication : 1 décembre 2009

Pagination : 228-230

ISBN : 978-2-9530779-4-0

ISSN : 0417-8726

\section{Référence électronique}

Denyse Rodriguez Tomé, "Yves Pauwels, Aux marges de la règle. Essai sur les ordres d'architecture à la Renaissance », Documents pour l'histoire des techniques [En ligne], 18| $2^{\mathrm{e}}$ semestre 2009, mis en ligne le 24 septembre 2010, consulté le 24 septembre 2020. URL : http://journals.openedition.org/dht/273 ; DOI : https://doi.org/10.4000/dht.273

Ce document a été généré automatiquement le 24 septembre 2020.

(c) Tous droits réservés 
Yves Pauwels, Aux marges de la règle. Essai sur les ordres d'architecture à la Renaissance

Wavre, Mardaga, 2008, 191 pages

Denyse Rodriguez Tomé

\section{RÉFÉRENCE}

Yves Pauwels, Aux marges de la règle. Essai sur les ordres d'architecture à la Renaissance, Wavre, Mardaga, 2008, 191 pages. 
1 Voici un ouvrage dévolu à l'architecture de la Renaissance en Italie et dans l'Europe de l'Ouest, qui porte un regard neuf sur la relation entre théorie et création tout au long du $\mathrm{XVI}^{\mathrm{e}}$ siècle. L'historien Yves Pauwels s'attache à démontrer comment les règles générales inspirées de l'Antiquité, énoncées notamment par Serlio, puis dans le Vignole, laissent une marge potentielle à l'invention de formes nouvelles. Cette grammaire architecturale définit la constitution des ordres et leurs proportions: à côté du dorique, de l'ionique et du corinthien, apparaissent le toscan, mais surtout le composite. Ce dernier ordre particulièrement encourage l'imagination des grands maîtres à Rome, à Florence, en France ou dans l'empire

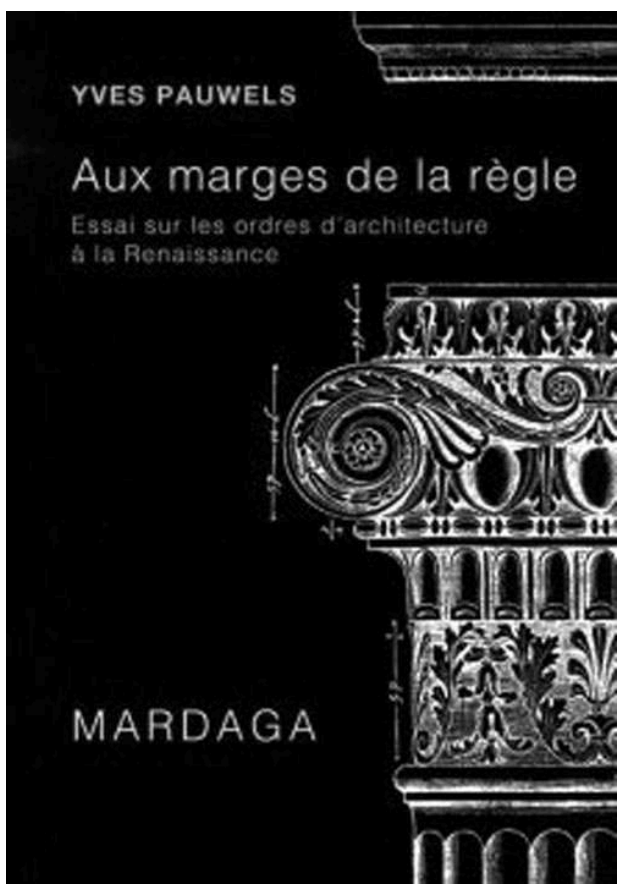
espagnol. Le mélange des styles est accepté et admiré en ce qu'il résulte du bon jugement de l'architecte, du ratio. Le composé domine dans la superposition des ordres, cette règle de composition qui place à l'étage supérieur l'ordre le plus élevé dans la hiérarchie des valeurs.

2 L'auteur démontre dans une première partie comment les théoriciens de la Renaissance mettent en ordre, selon leur conception de la réalité telle qu'elle doit être, les vestiges de l'Antiquité et les écrits de Vitruve. En adoptant un classement des informations par catégories stylistiques et en choisissant comme titres de chapitres les différents ordres ou colonnes, Serlio structure la diversité de l'héritage antique. Conciliant toutes ruines romaines et les descriptions de Vitruve, l'humaniste parvient à « embrasser tout l'art » de l'architecture. Par l'artifice d'un glissement sémantique, l'ordre universel de l'architecture est défini par les ordres d'architecture. Yves Pauwels livre une description des ordres helléniques assortie aux genres anthropomorphes vitruviens et à leurs appréciations par les architectes du Cinquecento : le dorique fort et trapu est masculin et viril, l'ionique plus élancé est féminin, assimilé à la matrone romaine ; plus fin et orné encore, le corinthien ressortit aux tendres vierges. Catégorie du «tout venant" absent chez Vitruve, le composite regroupe ces nombreux exemples archéologiques d'assortiments d'éléments doriques, ioniques et corinthiens, et plus particulièrement le quatrième ordre du Colisée, et relèverait alors d'une création romaine contemporaine de la nation victorieuse : il vient en bonne logique couronner les autres ordres.

3 Une deuxième partie de l'ouvrage est consacrée à l'abondante utilisation du composé moderne par les architectes de la Renaissance, amateurs de contrastes. Notamment, la technique du bossage de la pierre, inconnue de l'Antiquité, permet d'introduire du rustique dans la délicatesse d'une façade. Le composite italien concerne essentiellement l'œuvre de Michel-Ange, dont le génie inventif ne peut être classé dans la grammaire canonique sinon dans cette dernière catégorie à la fonction régulatrice. Vasari fait l'éloge de Michel-Ange : la théorie intègre la création contemporaine. 
4 En France où il s'établit à partir de 1541, Serlio introduit le composé romain dans ses édifices. Les artistes français qui professent ce renouveau esthétique lié à la Pléiade se nomment Jean Martin, Jean Goujon, Pierre Lescot, Philibert de l'Orme. Le mélange des styles se retrouve dans la Fontaine des Innocents à Paris avec ses chapiteaux composites, dans le décor de la grande salle du Louvre, ou encore au château d'Anet, où se mêlent éléments masculins et féminins, glose architecturale des amours du roi Henri II. La stratégie de Philibert de l'Orme théoricien étaye sa pratique architecturale : il consacre un livre entier de son Premier tome de l'architecture au composé, lieu par excellence du «comme il plaira». L'esthétique de la Renaissance associe donc à la tendance rationalisante qui met en ordre, un goût pour l'hybride et le monstrueux, le mélange et la variété.

5 Yves Pauwels soutient ainsi la première conclusion de son essai : la Renaissance a formalisé à la fois la règle et ses marges audacieuses, ces dernières ayant occupé dans la production architecturale une place de choix pour ces artistes inventifs. La troisième partie vient émettre le second volet de son analyse : le composé porte l'acte créateur à un niveau supranaturel. Les ordres précédents présentent l'éventail de l'expression de la nature humaine, dès lors le statut même de cet ordre supplémentaire, au-dessus des autres, ne peut être que sublime, et porte le triomphe du Prince. Les Entrées royales, équivalents modernes des Triomphes antiques, fournissent en bonne logique des ordres composites ou composés, par exemple en 1549, à Paris et à Gand, pour Henri II ou pour Charles Quint et son fils Philippe II d'Espagne.

6 Le composite est aussi lié au sublime et au divin par son rapport avec l'ordre du Temple de Jérusalem, dit ordre Salomonique. Les hommes de la Renaissance ont cru pouvoir définir plusieurs versions de cet ordre des ordres pour lors antérieur à l'Antiquité hellénique, inscrivant ainsi la supériorité de la culture judéo-chrétienne sur la culture païenne.

7 Enfin, l'ordre composite sert à l'établissement d'une architecture nationale, en Italie dans le Grand Duché de Toscane des Médicis, où le bossage de la pierre permet aussi de se revendiquer de l'ordre toscan rustique. Particulièrement, dans les deux principales puissances politiques prétendantes à l'hégémonie européenne, l'Espagne et la France, les architectes vont tirer à eux le composé et développer alors leur propre vocabulaire architectural. L'Espagne récemment reconquise adopte les colonnes balustres (le nom latin balaustium évoquant la grenade) et la composition corinthien/dorique. Les colonnes torses salomoniques et le décor de cep de vigne avec feuilles et raisins connaissent un succès éclatant à partir de 1600 jusque dans les colonies américaines. "Composées, modernes et françaises », les colonnes de Philibert de l'Orme empruntent aux traditions gothiques leurs piles de pierres et leur décor végétal à nervures. Le mélange de principes masculin et féminin, corinthien et dorique, relève d'un tempérament français conciliant la force, la gravité et la douceur, comme la fleur de lys, à la fois virginale et royale, grandeur débonnaire des monarques français face à la violence colérique de l'empereur espagnol.

8 À l'âge classique, en France, les qualités esthétiques du composite sont contestées, comme l'est le langage utilisé par les poètes de la Pléiade. La grandeur de la création artistique ne doit rien à l'invention de formes nouvelles, qui barbarisent l'Antique. Seuls les trois ordres helléniques ont droit de séjour. Le grand style de l'esthétique classique, voulue par Louis XIV, prétend fixer une civilisation glorieuse et immuable. L'esthétique de la nature finit par s'imposer aux artistes du siècle des Lumières. 
9 S'attachant tout autant sinon plus, aux écrits et publications qu'à la production architecturale, Yves Pauwels livre une analyse fouillée de nombreux auteurs et dessinateurs. Aux côtés de Serlio, Palladio, Scamozzi, Vasari, Guarini, Philibert de l'Orme, Jean Martin, Androuet du Cerceau, Fréart de Chambray, sont présents des auteurs espagnols tels que l'intéressant Sagredo, Jeronimo de Prado et Juan Bauttista Villapalpando, ou encore germanique ou flamand, Hans Blum et Bullant, qui permettent d'aborder la créativité dans l'empire espagnol. La rhétorique architecturale est pourvoyeuse d'idées autant que de modèles. Mêlant une passionnante étude littéraire à l'étude architecturale, Yves Pauwels nous fait remarquer dans le vocabulaire architectural de Philibert De l'Orme cette « inventiveté » de la langue de Ronsard ou de Rabelais.

D’une écriture agréable, doté d'une iconographie précise insérée dans le texte, cet ouvrage érudit, destiné aux spécialistes, pourrait être accessible à un public plus large, et ouvrir à une approche de l'architecture de la Renaissance et du Baroque par une démarche qui relie notamment l'histoire de l'architecture à l'histoire politique. C'est pourquoi on ne peut que regretter qu'à la différence des citations en latin, les abondantes citations en italien du Cinquecento ne soient pas traduites, même si l'idée principale en est généralement introduite par le texte de l'auteur.

\section{AUTEURS}

\section{DENYSE RODRIGUEZ TOMÉ}

CDHTE-Cnam 